\title{
Liens interorganisationnels
} et performance créaíne des agences de oleston en France

Ce papier cherche à expliquer la performance créative des organisations en fonction de leurs relations avec les institutions de leur champ organisationnel, les clients réputés et les partenaires professionnels. Une étude statistique est alors menée sur la performance créative de 87 agences de design en France en 2002. Cette performance est mesurée par trois indicateurs: la concentration des prix français, le nombre de prix étrangers et la diversité sectorielle du portefeuille marchand de l'agence. Les résultats montrent que la performance créative est une fonction croissante des relations avec les institutions et décroissante avec les clients réputés et les partenaires français. e développement de l'innovation occupe une
place importante en management stratégique
(Atamer et al., 2005). Dans ce sens, Woodman, Sawyer, Griffin (1993) rappellent que l'innovation engendre des changements organisationnels et que, pour les comprendre, il faut s'intéresser à la créativité, car elle engendre l'innovation. Ainsi, ces auteurs présentent la «créativité organisationnelle » comme une clé pertinente pour comprendre ces changements. Nous retenons, d'ailleurs, leur définition de ce concept: la création par des individus travaillant ensemble dans un système social complexe, d'un nouveau produit, service, idée, procédure ou processus. Quand cette création est valorisée et jugée utile, nous parlons alors de performance créative.

Qui, en revanche, joue le rôle de « juge »? Ford (1996) répond à cette question en se référant entre autres aux apports des théories néoinstitutionnalistes: il explique que l'action créative des organisations dépend de leur environnement composé des institutions étatiques et 
professionnelles ainsi que du marché des consommateurs. Par conséquent, l'environnement joue le rôle de « juge » et spécifie la valorisation et l'utilité de la création des organisations. Par exemple, la lumière électrique d'Edison a été jugée par les consommateurs comme aussi utile que la lumière au gaz et elle a été valorisée par les institutions telle que le district financier de Manhattan (Hargadon et Douglas, 2001).

Cet article s'intéresse donc à l'impact des relations interorganisationnelles sur la performance créative des organisations. Nous pensons, en effet, que les structures environnementales assimilées à des institutions (Scott 2001; Selznick, 1957) soutiennent la performance créative des organisations par leur « encouragement institutionnel » (Washington et Ventresca, 2004). Pour commencer à répondre à cette question, nous choisissons de nous inquiéter de l'influence de trois entités de l'environnement: les institutions étatiques et professionnelles, les clients réputés et les partenariats professionnels.

Par suite, l'objectif de cet article est d'enrichir les théories néo-institutionnalistes en prenant en compte l'action créative en management stratégique (Desreumaux, 2004). Dans cette optique, nous choisissons de travailler sur le champ du design industriel en France, car 1) cette profession est définie par son action créative, et 2) son travail créatif doit être valorisé et reconnu comme utile pour engendrer l'innovation sur le marché (Borja de Mozota, 2002; ministère de l'Industrie, 2002). C'est pourquoi, dans une première partie, nous présentons les hypothèses de recherche à partir de l'analyse de la littérature néoinstitutionnaliste. Nous détaillons, ensuite, la méthodologie utilisée pour tester ces hypothèses dans le cas des agences de design en France et indiquons, enfin, les résultats obtenus. Pour conclure, nous mettons en exergue quelques implications managériales pour les agences de design, les limites inhérentes à cette recherche, et des pistes de recherche.

\section{I. - LES INSTITUTIONS ET LA PERFORMANCE CRÉATIVE}

Le management stratégique appréhendé par les théories néoinstitutionnalistes emprunte les apports anciens d'économistes et de sociologues, comme Veblen, Weber, Parsons, mais il les adapte pour comprendre la réussite ou non des choix stratégiques d'une organisation (Desreumaux, 2004). En effet, les chercheurs institutionnalistes n'intègrent pas les intérêts des acteurs du champ (Scott, 2001). Ainsi, actuellement, l'approche néoinstitutionnaliste considère les institutions comme des sources d'isomorphisme (DiMaggio et Powell, 1983) mais également des soutiens aux stratégies émergentes (Washington et Ventresca, 2004), et les acteurs du champ organisationnel comme étant passifs face aux pressions institutionnelles, et actifs dans l'interprétation des normes (Oliver, 1991; Scott, 2001). En fait, ces évolutions nous permettent de mieux comprendre en quoi l'environnement institutionnel influence la performance créative d'une organisation (Ford, 1996), car elles montrent que ce qui est valorisé et considéré comme utile dépend des organisations et de leurs relations interorganisationnelles. C'est pourquoi, après avoir retracé les évolutions majeures de ce courant en lien avec la performance créative des organisations, le modèle et les hypothèses de recherche sont construits en vue d'être étudiés dans le 
champ organisationnel du design industriel en seconde partie.

\section{Les institutions : des sources d'isomorphisme et d'hétéromorphisme}

L'institutionnalisation d'un objet engendre la reconnaissance dudit objet. Il devient légitime; il est donc perçu comme désirable, adapté aux valeurs, normes et définitions du système social (Suchmann, 1995). La légitimité permet aux organisations d'assurer, en partie, leur survie, car elles sont comprises par les parties prenantes (Milliken et al., 1998; Suchmann, 1995). $\mathrm{Si}$, au début du processus, certaines structures adoptent volontairement les normes, valeurs ou règles du champ (Scott, 2001), ce n'est plus nécessairement le cas par la suite: les organisations utilisent des procédures, des structures ou adoptent des buts acceptés, pour prévenir la menace de leur légitimité (Elsbach, 1994).

Dans ce sens, DiMaggio et Powell (1983) cherchent à comprendre pourquoi les organisations d'un même champ organisationnel se ressemblent: ils souhaitent expliquer l'homogénéité et non la variation. Dans cette optique, ils utilisent le concept de « champ organisationnel »: un ensemble d'organisations opérant dans le même domaine, lui-même défini par la similarité de leurs services, produits ou fonctions. Le champ inclut aussi les organisations qui influencent de façon importante les autres entités: les fournisseurs, les clients, les acteurs régulateurs (État, associations professionnelles), les pourvoyeurs de financement. En outre, les champs organisationnels se forment autour de problèmes, et non de marchés ou de technologies (Hoffman, 1995).
DiMaggio et Powell (1983) constatent que l'augmentation des interactions entre les organisations d'un même champ et l'émergence de structures interorganisationnelles dans ledit champ expliquent en partie l'homogénéité des organisations. Nous parlons alors d'isomorphisme comme d'un processus qui force une unité de la même population à ressembler aux autres unités qui font face aux mêmes conditions environnementales. Le résultat de ce processus est l'homogénéité des organisations d'un champ. En fait, dans un contexte d'aversion au risque, l'isomorphisme apparaît comme le comportement optimal (Desreumaux, 2004).

Des études ont opérationnalisé les différents types d'isomorphisme (Mizruchi et Fein, 1999). L'isomorphisme coercitif trouve notamment un développement dans l'étude menée par Edelman et al. (1999), qui s'intéressent aux relations des organisations avec leur personnel concernant l'égalité devant l'emploi. L'isomorphisme normatif est illustré, entre autres, dans le travail de King et Lenox (2003) : ils veulent comprendre l'auto-régulation des industries comme complément à la régulation gouvernementale, dans le cas des entreprises de chimie. Ils s'inquiètent alors de l'appartenance ou non de ces entreprises à l'association officielle de leur industrie. Enfin, l'isomorphisme mimétique est étudié, par exemple, par Schoonhoven, Eisenhardt et Lyman (1990) : ils travaillent sur la vitesse de développement d'un produit par des nouvelles firmes dans un marché. Les études montrent ainsi que face à l'incertitude, les organisations adoptent les mêmes stratégies que les autres, d'où leur ressemblance. 
Pourtant, même dans ce contexte, des organisations n'adoptent pas le comportement institutionnalisé. En effet, Oliver (1991) montre que certaines essaient de concilier les différentes pressions institutionnelles, de négocier avec les acteurs institutionnels, tandis que d'autres se détachent des normes en les évitant ou en déguisant leur conformité. Oliver (1991) développe alors l'idée selon laquelle les organisations ne sont pas passives: elles optent pour d'autres stratégies de réponses face aux pressions isomorphiques des institutions, comme le compromis, l'évitement, la mise au défi et la manipulation.

Ces organisations sont de ce fait considérées comme des « renégats » (Kondra et Hinings, 1998). Elles sont en dehors des normes institutionnelles par choix ou par distorsion génétique (Hannan et Freeman, 1986 et 1989 in Kondra et Hinings, 1998). Les organisations qui adoptent ces normes peuvent alors 1) contraindre les renégats à se soumettre aux pressions isomorphiques, 2) les imiter ou 3) les ignorer. Le comportement des renégats introduit, par conséquent, le changement et perturbe l'adoption des valeurs de la logique dominante du champ. S'il est, par suite, considéré comme un atout dans l'accès à un niveau de performance avantageux, ce comportement « renégat » initialement devient valorisé par les acteurs du champ.

Néanmoins, bien que l'approche des institutions comme des sources d'isomorphisme soit riche d'enseignements, elle est insatisfaisante sur une question-clé du management stratégique: le développement de l'innovation et de la créativité dans les organisations (Revue française de gestion, 2005). En effet, le postulat selon lequel les institutions sont des sources d'isomorphisme entraîne une considération négative des organisations en dehors des normes institutionnelles: elles sont des « renégats ». Or, la littérature néo-institutionnaliste reconnaît l'importance de la diversité pour les organisations ce qui passe par la critique de ces normes (Farjoun, 2002; Lounsbury, 2001; Kondra et Hinings, 1998).

Par exemple, Greve et Taylor (2000) explorent les situations où des innovations génèrent une réponse non mimétique, comme dans le cas du réseau radiophonique américains. Ils concluent que les innovations changent les conditions managériales sur lesquelles les actions sont possibles et bénéfiques. En effet, elles encouragent le changement en suggérant de nouvelles opportunités et les rendent acceptables aux yeux des organisations. En revanche, une condition doit être remplie pour arriver à une prise en compte positive de la discussion des normes institutionnelles : les managers doivent être liés à la communauté. Ainsi, leurs relations avec d'autres organisations facilitent la critique susceptible d'engendrer une innovation.

Or, selon DiMaggio et Powell (1983), ces mêmes relations orientent les organisations vers l'isomorphisme. Ces deux conclusions ne sont pas paradoxales si nous considérerons les institutions comme des soutiens à la différence ou des forces hétéromorphiques: elles encouragent les organisations d'un champ à être différentes des autres. Cette conception s'apparente à celle de Washington et Ventresca (2004) qui ne veulent pas réduire les institutions à des « cages de fer ». Ces auteurs proposent, dans ce sens, le concept « d'encouragement 
institutionnel » : les institutions établissent les conditions du changement et les éléments légitimes à son incorporation. Washington et Ventresca (2004) détaillent, par ailleurs, trois mécanismes favorisant cet encouragement: 1) l'existence d'une logique dominante soutenant l'incorporation de stratégies émergentes, 2) les liens de l'organisation avec les institutions du champ et 3) l'intégration de la stratégie émergente par d'autres organisations.

L'étude de Rao, Monin et Durand (2003) sur les chefs de la cuisine française illustre ce concept. Les auteurs rappellent les « Dix commandements de la nouvelle cuisine » promulgués par deux journalistes culinaires Henri Gault et Christian Millau; le dernier commandement enjoint à « Tu dois être inventif ». Les quatre valeurs fondamentales de la nouvelle cuisine sont: vérité, légèreté, simplicité et imagination. De ce fait, les normes de la cuisine traditionnelle ont été critiquées, ce qui a encouragé le changement culinaire. Les institutions telles que la presse ont valorisé les chefs assimilés initialement à des « renégats », et ont légitimé leur esprit créatif. Cet encouragement perdure à travers notamment le Guide Michelin qui légitime par des étoiles la performance créative des chefs. Par voie de conséquence, les institutions exercent une pression à la différence: les chefs doivent proposer des plats nouveaux. Le résultat d'un tel processus est l'hétérogénéité des organisations d'un champ.

De ce fait, la performance créative d'une organisation peut être soutenue par les relations interorganisationnelles institutionnelles. En adoptant l'approche de Selznick (1957), nous considérons les organisations comme jouant le rôle d'institution, car à même d'établir des normes, des lois, des valeurs à respecter. Par conséquent, le modèle de recherche tient compte des relations entre une organisation et les institutions étatiques et professionnelles d'un champ organisationnel, mais aussi entre l'organisation et ses clientes et ses partenaires professionnels.

\section{Modèle conceptuel et hypothèses de recherche}

Les études empiriques s'appuyant sur les théories néoinstitutionnalistes précisent l'influence des entités du champ sur les décisions stratégiques des organisations, y compris sur la volonté de l'organisation à créer un produit, un service, une idée valorisable et utile (Woodman et al., 1993). Comme premier facteur explicatif de la performance créatif, nous retenons alors « les institutions étatiques et professionnelles », comprenant l'ensemble des entités du champ dont les objectifs ne sont pas la rentabilité économique, mais le soutien et la défense de la profession, tels que les syndicats et les associations de promotion.

Dans cette optique, Washington et Ventresca (2004) expliquent que les liens avec les institutions étatiques et professionnelles, qui soutiennent l'incorporation de stratégies émergentes, encouragent les organisations à incorporer à leur tour ces stratégies. De ce fait, si les institutions soutiennent la critique des normes institutionnelles, les organisations sont soutenues positivement dans leurs décisions d'être créatives par la critique. Par exemple, Van den Bulte et Lilien (2001) cherchent à savoir comment la communauté médicale a créé une nouvelle manière de soigner en adoptant les 
antibiotiques. Ils tiennent compte alors de son exposition aux revues médicales, où des pages de publicité vantent les mérites des antibiotiques. Lorsque Durand et al. (2005) s'intéressent à la créativité des chefs « étoilés » de la cuisine française, ils tiennent compte notamment de leurs évaluations passées et donc de leurs liens avec d'autres institutions (Meilleur Ouvrier de France, le Prosper Montagné ou le prix Taittinger).

$\mathrm{Si}$ ces études sous-entendent un impact positif sur la performance créative des relations de l'organisation avec les institutions du champ, Amabile (1988) souligne qu'un environnement comprenant de nombreuses contraintes et des critères d'évaluations irréalistes ou inadaptés freine la créativité dans une organisation. Pareillement, si les individus se sentent contrôlés par l'extérieur, leur créativité est entravée (Amabile et al., 1996). Ces travaux supposent alors un impact négatif de nombreuses relations organisationnelles avec les institutions du champ.

Or, dans cet article, nous considérons les institutions comme des soutiens aux stratégies émergentes, nous pensons alors que l'impact de ces relations interorganisationnelles sur la performance créative est positif. En outre, les études de Edelman et al. (1999), de Gerber (2002) ou encore de Milliken et al. (1998) tiennent compte des influences géographiques sur leur objet d'étude. Greve et Taylor (2000) précisent que les innovations d'une région géographique augmentent leur intégration par les autres organisations du champ de la même région et du pays et non celles de régions plus lointaines, voire étrangères.
Hypothèse 1. La performance créative d'une organisation est une fonction croissante de la multiplicité de ses relations avec les institutions étatiques et professionnelles nationales.

De plus, Ford (1996) explique que l'action créative dépend des clients, assimilés à des institutions (Selznick, 1957) quand ils jouent un rôle dans l'acquisition et le maintien de la légitimité d'une organisation. D'ailleurs, Zuckerman (1999) souligne l'importance de prendre en considération le public visé par la communication de la légitimité. En effet, selon lui, sans un auditoire, la légitimité perd sa valeur et sa signification. Les organisations sont considérées alors comme des candidats, qui se présentent à un auditoire pour gagner sa faveur. En outre, Benjamin et Podolny (1999) précisent que les clients réputés sont des organisations, qui apportent leur crédit aux décisions stratégiques des organisations, ce qui est également le cas lors de stratégies émergentes. De ce fait, les organisations réputées influencent la performance créative.

Par exemple, Baker et al. (1998) cherchent à comprendre le fonctionnement d'un marché à partir, notamment, des théories néoinstitutionnalistes. Ils prennent pour terrain les relations entre des clients et des agences de publicités. Ils spécifient alors cette relation en décrivant le client: quelle est son histoire ? A-t-il subi un rachat ou en est-il à l'origine? L'étude de ces dépendances se retrouve dans le travail de Jones et al. (2002), qui étudient la construction de la légitimation des architectes et détaillent la relation de ces acteurs avec leurs clients. Ils citent la remarque de Benoit-Smullyann (1944 in Jones et al., 2002) : «Ceux qui 
s'associent avec des gens de grand prestige participent à ce prestige ».

Ils indiquent, néanmoins, une conclusion de Blau (1979 in Jones et al., 2002) : des liens forts avec des clients majeurs inhibent la capacité d'innovation des entreprises. En effet, la réputation est synonyme d'avantage concurrentiel, que veulent préserver les organisations réputées. Or, l'ambiguité leur nuit: elle peut ne pas leur permettre de maximiser leur statut social (Fombrun et Shanley 1990). De plus, les managers doivent justifier rationnellement leurs décisions stratégiques, comme de créer un nouveau produit, pour échapper à une éventuelle éviction en cas d'échec (Elsbach, 1994) : ils préfèrent limiter l'ambiguïté susceptible d'être générée par de nouveaux produits, idées, services... C'est pourquoi, nous pensons que les organisations en relation avec des clients réputés s'investissent moins dans la créativité, d'où un impact négatif sur leur performance créative.

Hypothèse 2. La performance créative d'une organisation est une fonction décroissante de la multiplicité de ses relations avec des clients réputés.

Le dernier facteur pris en compte dans le modèle concerne les partenaires professionnels : il regroupe l'ensemble des organisations à but lucratif en partenariat avec l'organisation étudiée et dont la pratique est complémentaire à ladite organisation. Un partenariat peut être ponctuel sur un projet ou durer dans le temps. À l'instar des organisations réputées, les partenaires sont aussi des institutions (Selznick, 1957) et ils influencent la performance créative d'une organisation.

En revanche, comme le soulignent Pfeffer et Salancik (1978), un projet avec plus de spécialistes crée une interdépendance entre eux, d'où une relation perdurant au fil des années. Baum et Korn (1999) précisent, cependant, que l'interdépendance des deux firmes leur demande d'accepter leur supériorité respective dans leur propre domaine d'activité. La vision transversale d'un problème permet, par conséquent, d'accroître la créativité de l'action engagée.

En effet, Ford (1996) explique que l'action créative dépend de trois facteurs :

1) la compréhension (sensemaking) : recherche d'information, orientée par ses propres schémas cognitifs;

2) la motivation: il s'agit des objectifs personnels, fixés par les croyances de l'individu en ses capacités (confiance en soi), par l'accueil de l'environnement pour l'action envisagée et par ses émotions ;

3) les savoir-faire et les compétences: cela regroupe les connaissances de l'individu sur le domaine, ses aptitudes comportementales, son réseau social, et sa propension à penser différemment. Ainsi, en créant une relation privilégiée avec d'autres professionnels, cela démultiplie les connaissances, les sources d'informations, les talents, etc.

Aussi, comme l'expliquent Drazin et al. (1999), la créativité émerge d'un processus de négociation entre les intérêts multiples de plusieurs communautés de l'organisation. Or, selon eux, dans une organisation, trois niveaux d'analyse peuvent être retenus : intrasubjectif, intersubjectif et collectif. In fine, le sens donné au résultat est le fruit d'un compromis politique entre communautés. Par conséquent, associer des partenaires au travail créatif d'une organisation correspond au choix d'agrandir le nombre de communautés impliquées dans le compromis en vue de gagner en légitimité auprès des professionnels déjà reconnus, 
mais aussi d'introduire des perturbations dynamisantes (Drazin et al., 1999). De ce fait, les organisations font appel à leurs partenaires pour accroître le nombre de regards obliques sur un même projet créatif, ce qui a un impact positif sur sa performance créative. Comme dans le cas des relations avec les institutions, nous nous intéressons aux relations interorganisationnelles nationales (Greve et al., 2000).

Hypothèse 3. La performance créative d'une organisation est une fonction croissante de la multiplicité de ses relations avec les partenaires nationaux.

$\mathrm{Ce}$ jeu d'hypothèses nous permet alors de proposer le modèle conceptuel de la recherche pour expliquer la performance créative des organisations par leurs relations avec d'autres organisations du champ organisationnel (figure 1). Ce modèle est développé dans la seconde partie dans l'étude des performances créatives des agences de design en France.

\section{II. - LES AGENCES DE DESIGN EN FRANCE : UNE PERFORMANCE CRÉATIVE ORIENTÉE PAR LES INSTITUTIONS}

Si le design industriel a émergé en Europe avec la révolution industrielle du $\mathrm{XIX}^{\mathrm{e}}$ siècle (Lucie-Smith, 1983), la France intègre réellement cette discipline créative dans ses pratiques organisationnelles après la Seconde Guerre mondiale (De Noblet, 1988; Guidot, 2000). Depuis cette époque, nous assistons à une montée en puissance des actions des institutions en vue de promouvoir toujours davantage le design industriel (Meyer, 2001), comme l'inauguration du Centre de création industrielle par le président Georges Pompidou en 1969, l'ouverture de l'École nationale supérieure de création industrielle par le ministre Jack Lang en 1981 ou prochainement la Cité du design par le sénateur-maire de Saint- Étienne Michel Thiollière.

Les institutions du champ organisationnel soutiennent alors la performance créative des entités centrales du champ, à savoir les

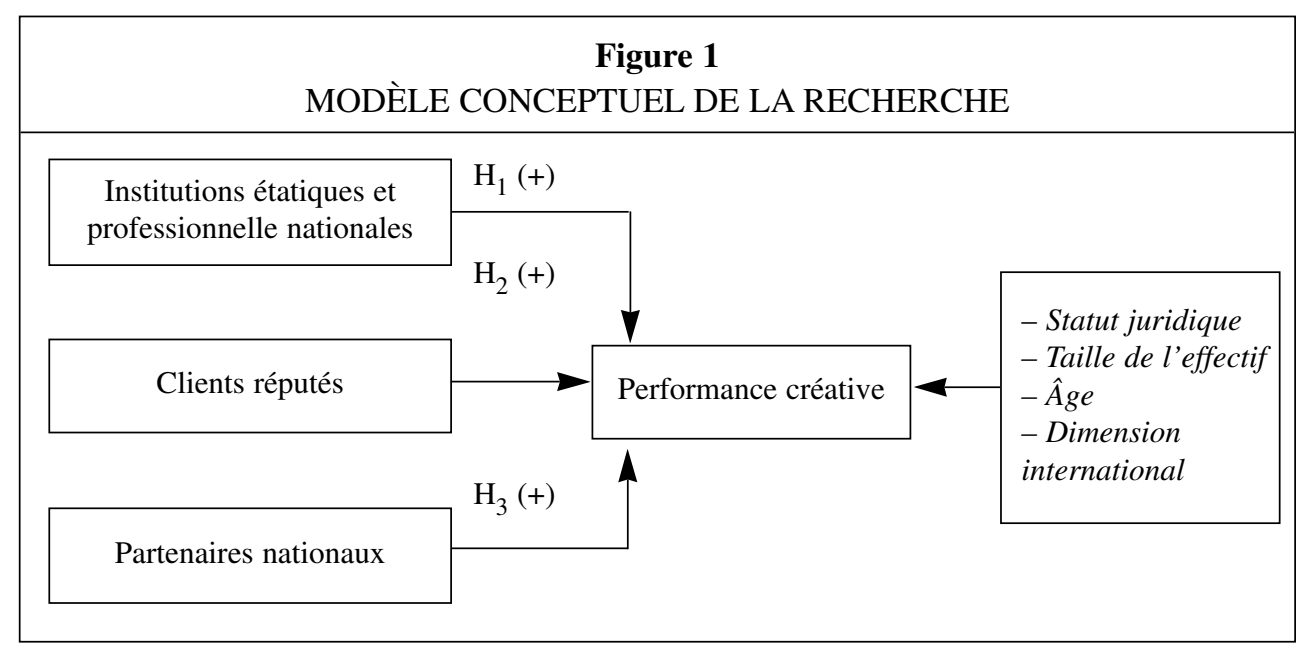

Remarque: sont intégrés les facteurs de contrôle pris en compte dans les tests statistiques dans le cas de la performance créative des agences de design. 
agences de design. Elles les encouragent à créer des produits, des services, des idées divergents, mais qui soient valorisables et utiles. En effet, le design industriel crée de la différence dans un marché, mais cette différence doit être compréhensible par les consommateurs (Borja de Mozota, 2002).

Ainsi, par design industriel, nous entendons: « activité créatrice dont le but est de déterminer les multiples facettes des objets, processus, services » $\left(\mathrm{ICSID}^{1}, 2000\right)$, mais aussi une discipline créative en charge de la production en série des signes émis par une organisation ${ }^{2}$. Les designers sont donc des spécialistes en conception formelle qui jouent le rôle de médiateur entre l'utilisateur et le fabricant (Borja de Mozota, 2002; Endt, 1990). Une agence de design est une organisation comprenant au moins un designer industriel et dont le métier est la conception industrielle dans les différentes pratiques du design: le design graphique, le design produit, le design environnemental ou architectural, le design sensoriel et le design management.

Eu égard aux développements théoriques précédents, nous nous focalisons sur la performance créative des agences de design. C'est pourquoi, après avoir relaté des repères de compréhension dans le champ organisationnel du design industriel en France, nous cherchons à expliquer leur performance créative en fonction de leurs relations interorganisationnelles.

\section{Points de repère dans le champ organisationnel}

Selon Suchmann (1995), un objet, comme le design industriel, doit être institutionnalisé pour être perçu comme légitime. Or, Scott (2001) précise que l'institutionnalisation d'un tel objet suppose l'action de quatre acteurs-clés. La puissance publique joue plusieurs rôles. Elle émet des lois dites coercitives, car incontournables.

\section{DÉFINITION DES SIGLES UTILISÉS}

ACPI : Agence de promotion de la création industrielle ; www.apci.asso.fr

CDRA : Centre de design du Rhône-Alpes ; www.cdra.asso.fr

CNDP : Centre national de documentation pédagogique ; www.cndp.fr

DAP : Délégation aux arts plastiques

http://www.culture.gouv.fr/culture/dap/dap/index-dap.html

DARPMI : Direction de l'action régionale et de la petite et moyenne industrie

ENSCI : École nationale supérieure de la création industrielle ; www.ensci.com

FEDI : Fédération des designers industriels

www.designfrance.tm.fr/designers/groupements-professionnels/design-industriel.html

ICSID : International Council of Societies of Industrial Design ; www.icsid.org

OPQDI : Office professionnel de qualification des designers industriels

UFDI : Union française des designers industriels

1. L'ICSID est un organisme qui regroupe sur le plan international les différents syndicats professionnels de designers. 2. On parle du «Design graphique » pour les signes en 2D comme les couleurs, le graphisme sur un packaging, le logo, la signalétique, etc.; puis du « Design produit » pour les signes en 3D comme le travail sur le volume, les formes; et, enfin, du « Design environnement » pour les signes architecturaux comme l'aménagement d'un linéaire, d'un stand, d'un magasin, etc. 
Elle régule, contrôle et juge les actions de tous. Ses sanctions sont légitimes et légales. Dans le champ du design industriel, nous trouvons les départements des ministères de l'Industrie (DARPMI) et de la Culture (DAP), mais aussi les formations universitaires, comme l'ENSCI, Dual design et les pôles design du CNDP (voir encadré pour la signification des sigles).

Les professionnels exercent, quant à eux, un contrôle via des processus normatifs et culturels. Ils définissent les cadres « ontologiques » de leur profession à travers, notamment, des codes de déontologie, des certifications... Pour le design industriel, nous trouvons principalement le syndicat FEDI qui n'est pas encore significatif, car il ne regroupe pas assez de professionnels.

Les organisations et associations diffusent des mécanismes culturels dans le système économique, tout en étant reconnues non pas comme des ersatz des nations États, mais comme des institutions capables d'influencer les décisions étatiques. Le champ du design industriel en France comprend notamment deux grandes associations: le CDRA à Lyon et l'APCI à Paris. Elles ont en charge la promotion du design par des conférences, des guides comme «Panorama Design », des formations spécifiques aux designers ou aux entrepreneurs.

Enfin, les cadres culturels donnent un sens aux objets, qui sont compris et reconnus grâce à leurs référents culturels et cognitifs. La production d'objets transforme également l'environnement en créant de nouveaux cadres culturels. Nous retrouvons dans cette catégorie toutes les actions de diffusion du design industriel par les musées d'art moderne, la Biennale internationale du design à Saint-Étienne et les expositions de l'APCI à La Villette à Paris. Les objets sont ainsi légitimés par leur exposition dans des lieux légitimes.

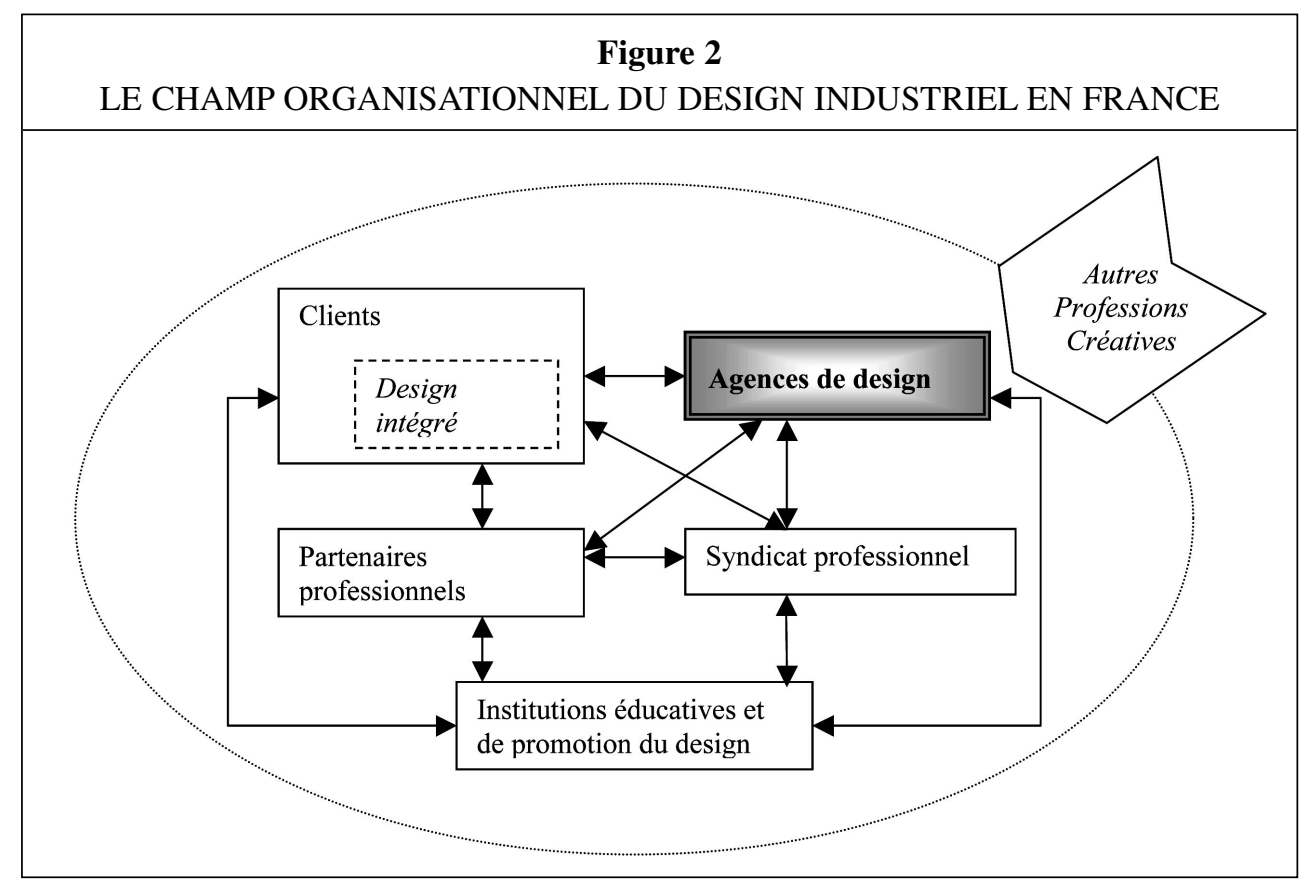


La figure 2 propose une représentation possible du champ organisationnel du design industriel en France, où apparaissent les acteurs de l'institutionnalisation et les entités incluses dans le modèle de la recherche.

\section{Méthodologie de recherche et résultats}

Cette section a pour but d'exposer nos choix en matière de construction des variables. Cela nous permet de signifier des limites inhérentes aux choix méthodologiques et donc de limiter la portée des résultats (Drucker-Godard et al. in Thiétart, 2003).

\section{Sources des données}

Notre étude s'appuie sur des données secondaires : la revue de l'éditeur Stratégies Le guide des professionnels du design de l'année 2002. Nous avons choisi cette revue en raison de sa représentativité en matière d'expertise en design - présence des différents types de design -, de ses frontières temporelles et de la taille de son auditoire, à savoir 7000 personnes (directeurs marketing, de communication, dirigeants et directeur de création des agences de design, etc.). En 2004, nous avons complété les données de la revue par celles disponibles sur les sites Internet des agences et sur le site societe.com. Notre échantillon regroupe 87 agences de design différentes. Les avantages des sources secondaires rédigées par les organisations elles-mêmes sont multiples (Baumard et al. in Thiétart, 2003). Nous n'intervenons pas dans le recueil des données: l'agence dit ce qu'elle veut communiquer. De plus, le format de l'ensemble des descriptions des agences est constant. En effet, chaque agence reçoit un formulaire standard à remplir, ce qui l'inci- te fortement à renseigner les mêmes catégories. Nous avons des données sur la personne morale comme ses coordonnées, sa date de création, la structure de son capital, sa forme juridique, son effectif, les noms des dirigeants, la description de son portefeuille commercial, ses relations avec des institutions du champ, l'existence de partenariats professionnels, et le détail des récompenses reçues.

Toutefois, une limite importante à l'exploitation des données réside dans le caractère commercial de la revue: elle est payante et la parution d'une agence est fonction de sa gestion financière. De plus, la description des agences a pour but d'attirer le futur client: cette publication rentre dans une logique de stratégie de communication. Ainsi, les données secondaires dessinent un type précis de comportement de l'agence, considéré, toutefois, comme véridique. Nous devons donc prendre en considération ces limites lors de la discussion des résultats. Elles peuvent éventuellement expliquer l'absence de signification de certains liens hypothétiques, mais aussi relativiser certains liens conjecturaux. Une dernière limite réside dans l'absence de données qualitatives obtenues par entretien: cela nous aurait permis de comprendre, en fonction des acteurs concernées (le designer, le commercial, etc.), quel est l'impact sur la performance créative des agences de leurs relations interorganisationnelles.

\section{Construction et description des variables}

Pour rendre opératoire le modèle de recherche, nous avons décliné les concepts en variables. Au final, nous en avons dix, en incluant des variables de contrôle (cf. tableau 1). En raison de la subjectivité inhérente au concept de « performance 
créative », nous n'avons pas réduit la mesure à un seul indicateur (Lazersfeld, 1965). Dans le cas particulier de la performance créative des organisations étudiées, à savoir les agences de design, trois indicateurs servent à mesurer le concept. Tout d'abord, nous tenons compte de la concentration des prix cités. Il s'agit avant tout d'une performance créative professionnelle. Dans le champ organisationnel du design industriel français, il existe cinq grandes récompenses (cf. encadré ci-après) donnés par des représentants des entités du champ (des designers en agence ou intégrés, des professeurs, des clients industriels, etc.). Cela signifie que les créations récompensées sont reconnues par d'autres professionnels comme des «produit créatifs ». Ainsi, nous mesurons 1) la spécialisation de l'agence dans chacun de ces cinq prix: (nombre de récompenses citées dans la catégorie)/(nombre total de prix cités); et 2) la concentration de ces prix par la somme des spécialisations au carré.

\begin{tabular}{|c|c|}
\hline \multicolumn{2}{|r|}{$\begin{array}{c}\text { Tableau } 1 \\
\text { LES GRANDS PRIX FRANÇAIS RETENUS DANS L'ÉTUDE }\end{array}$} \\
\hline Prix & Description \\
\hline $\begin{array}{l}\text { Le Janus de } \\
\text { l'industrie }\end{array}$ & $\begin{array}{l}\text { Depuis l'après-guerre, l'Institut français du design (IFD), observatoire de la création } \\
\text { industrielle entre les industriels et les créateurs, organise ce prix, qui vise les } \\
\text { produits industriels dont les qualités fonctionnelles, techniques et esthétiques sont } \\
\text { jugées conformes aux critères théoriques et pratiques du design industriel. }\end{array}$ \\
\hline L'Oscar & $\begin{array}{l}\text { Depuis la moitié des années 1950, le Groupe industrie service info (GISI) } \\
\text { organise les oscars de l'emballage avec, aujourd'hui, L'Usine Nouvelle, LSA et } \\
\text { Emballages Magasine. Trois catégories existent: les oscars en distribution, en } \\
\text { production et en consommation. Ce prix récompense le design packaging. } \\
\text { http://www.oscardelemballage.com/ }\end{array}$ \\
\hline $\begin{array}{l}\text { Les prix de } \\
\text { Stratégies }\end{array}$ & $\begin{array}{l}\text { Depuis la moitié des années } 1980 \text {, le groupe Stratégies organise des prix du } \\
\text { design pour récompenser plusieurs domaines de design: design graphique, } \\
\text { design produit, design d'environnement, design global. } \\
\text { http://www.strategies.fr/gdprix/ }\end{array}$ \\
\hline Le TopCom & $\begin{array}{l}\text { En 1999, Patrice Legendre crée les prix TopCom au sein du congrès TopCom } \\
\text { existant depuis 1995. Le prix prime les réalisations des professionnels de la } \\
\text { communication. Plusieurs sections existent, dont celle du design, où sont évalués } \\
\text { le logo, l'application visuelle, la charte éditorial, les sites Internet, packaging, } \\
\text { architecture commerciale, merchandising. } \\
\text { http://www.topcom.fr/indexl.htm }\end{array}$ \\
\hline L'Observeur & $\begin{array}{l}\text { Depuis 1999, l'APCI organise ce prix qui récompense des produits innovants. Le } \\
\text { jury décerne également des « étoiles » aux produits qui respectent différentes } \\
\text { contraintes, comme une démarche éco-design, la sécurité, l'ergonomie, l'approche } \\
\text { sensorielle... L'ANVAR, l'INPI et l'ADEME attribuent aussi leur label pour } \\
\text { distinguer certains produits remarquables dans leur domaine. } \\
\text { http://www.apci.asso.fr/l_observeur/decouvrez_observeur.php }\end{array}$ \\
\hline
\end{tabular}


Ensuite, nous intégrons la dimension internationale de l'agence par le nombre de prix étrangers cités, mesurés par le logarithme du nombre de prix étrangers cités. Cet indicateur nous permet d'éviter de tenir compte uniquement des reconnaissances francofrançaises, parfois critiquées par le manque de diversité de la composition des jurys.

Enfin, nous tenons compte de la diversité sectorielle du portefeuille commercial de l'agence de design. Il s'agit alors d'une performance commerciale. En effet, nous considérons une agence comme créative si elle est capable de travailler sur divers projets en design industriel. Sa performance créative réside alors dans sa capacité à s'adapter à des contraintes distinctes selon des univers aussi différents que celui de

\begin{tabular}{|c|c|}
\hline \multicolumn{2}{|r|}{$\begin{array}{c}\text { Tableau } \mathbf{2} \\
\text { COMPOSITION DES TREIZE SECTEURS DE L'ÉTUDE }\end{array}$} \\
\hline Nom du secteur & Activités du secteur \\
\hline Banques & Compagnie d'investissement, groupe de crédit. \\
\hline Assurances & Mutuelle, assurance spécialisé. \\
\hline $\begin{array}{l}\text { Distribution de } \\
\text { biens de } \\
\text { consommation }\end{array}$ & $\begin{array}{l}\text { Distribution spécialisée, Vente par correspondance, fournitures de bureaux, } \\
\text { distribution de meubles, de vêtements, de chaussures, d'articles de sport, } \\
\text { d'électroménager... }\end{array}$ \\
\hline Technologie & $\begin{array}{l}\text { Hardware, software, équipement électronique, électrique, informatique, high- } \\
\text { tech, défense, optique. }\end{array}$ \\
\hline $\begin{array}{l}\text { Ressources et } \\
\text { Industries }\end{array}$ & $\begin{array}{l}\text { Tabac, industries textiles, sidérurgie, pétrole, emballage, aluminium, verre, } \\
\text { papier, énergie. }\end{array}$ \\
\hline $\begin{array}{l}\text { Chimie « pour } \\
\text { les hommes » }\end{array}$ & $\begin{array}{l}\text { Produits pharmaceutiques (laboratoire), hygiène, entretien, cosmétiques, } \\
\text { parfum. }\end{array}$ \\
\hline Agroalimentaire & $\begin{array}{l}\text { Alimentation, produits laitiers, vins, sucrerie, spiritueux, nutrition animale, } \\
\text { salaisons, conserves de légumes, biscuiterie, surgelés, café, chocolat, céréales. }\end{array}$ \\
\hline $\begin{array}{l}\text { Moyens de } \\
\text { locomotion }\end{array}$ & $\begin{array}{l}\text { Automobile, aérospatiale, aéronautique, équipements automobiles, } \\
\text { pneumatiques, moteurs. }\end{array}$ \\
\hline Constructions & BTP, équipements de construction. \\
\hline Communication & Média (TV, presse, radio, Internet, téléphonie), photographie, publicité. \\
\hline Détente & $\begin{array}{l}\text { Hôtellerie, restauration, jeux, activités culturelles, sportives, agences de } \\
\text { voyages, tourisme. }\end{array}$ \\
\hline $\begin{array}{l}\text { Services aux } \\
\text { collectivités }\end{array}$ & $\begin{array}{l}\text { Distribution de gaz, d'électricité, du courrier, de l'eau, services aux entreprises } \\
\text { (juridique, conseil, recherche, travail temporaire, etc.), contrôle. }\end{array}$ \\
\hline Transports & Transport aérien, terrestre, maritime, ferroviaire, transport urbain. \\
\hline
\end{tabular}


l'agroalimentaire, de la banque, de la construction ou des services publics. Nous avons classé tous les clients cités par l'agence en fonction de treize secteurs différents. Puis, nous avons calculé la spécialisation de l'agence dans chaque secteur: nombre de clients cités dans le secteur/nombre total de clients cités. Enfin, nous avons mesuré l'indice d'Herfindhal par le calcul (1-somme des spécialisations au carré).

Les variables indépendantes ne sont mesurées que par un seul indicateur. En matière de design industriel en France, il existe quelques institutions professionnelles fréquemment citées par les agences, comme l'APCI, l'association design communication (ADC), l'IFD, l'Association pour la valorisation de l'innovation dans l'ameublement (VIA), l'UFDI devenue depuis 1999 la FEDI, l'OPQDI, l'ICSID. Les relations interorganisationnelles de l'agence avec les institutions étatiques et professionnelles françaises sont alors mesurées par le logarithme du nombre de relations citées par l'agence.

Pareillement, les relations avec des partenariats français sont mesurées par le logarithme du nombre de partenariats cités. Par exemple, en 2002, l'agence Barré \& Associés annonce un partenariat avec ReadMark à Limonest, qui propose des produits-clés en main et des analyses marketing de diversification produit. L'agence Coconuts a un partenaire pour les activités de photographie argentique et numérique, Shogun à La Madeleine.

Dans le cas des clients réputés, nous avons comptabilisé, pour l'année 2002, le nombre de clients appartenant à l'indice boursier de la place de Paris, le CAC 40, et le nombre de leaders français et mondiaux cités. Les leaders ont été identifiés pour les treize secteurs crées pour l'étude à partir du classement des entreprises françaises et mondiales de la revue L'Expansion. Nous avons estimé que si un client est à la fois au CAC 40, leader français et mondial, le client est plus réputé que le client « simplement » au CAC 40. De ce fait, l'indicateur retenu est: le logarithme de la somme du nombre de clients du CAC 40, de clients leaders français et internationaux.

Nous avons retenu également des variables de contrôle: son statut juridique (1 pour une société anonyme, 0 sinon), la taille de l'agence (logarithme de l'effectif), son âge (logarithme du nombre d'années qui court entre la création de l'agence et 2002), et sa dimension internationale (oui $=1$, non $=0$ ).

\section{Tests et résultats}

Le modèle statistique retenu est une régression linéaire multiple: la variable dépendante peut être calculée comme une fonction linéaire d'un ensemble spécifique de variables indépendantes plus un terme d'erreur. Nous supposons que chaque observation de l'échantillon est caractérisée par l'équation suivante: $\mathrm{y}=\mathrm{x}_{1} \mathrm{~B}_{1}+\mathrm{x}_{2} \mathrm{~B}_{2}+\ldots+$ $\mathrm{x}_{\mathrm{k}} \mathrm{B}_{\mathrm{k}}+$ Erreur (Greene, 2003). Comme les trois indicateurs ne sont pas corrélés, nous conservons la mesure de la performance créative à partir de ces indicateurs : chaque modèle statistique est décliné en fonction d'eux.

Pour tester chaque hypothèse, nous avons tout d'abord bâti le modèle de base, où sont prises en compte les variables de contrôle: le statut juridique, la taille, l'âge et la dimension internationale. Nous constatons 


\begin{tabular}{|c|c|c|c|}
\hline \multicolumn{4}{|c|}{$\begin{array}{c}\text { Tableau } 3 \\
\text { RÉCAPITULATIF DES INDICATEURS DE MESURE DE CHAQUE VARIABLE }\end{array}$} \\
\hline Variables & $\begin{array}{l}\text { Indicateur } \\
\text { de mesure }\end{array}$ & Variables & $\begin{array}{l}\text { Indicateur } \\
\text { de mesure }\end{array}$ \\
\hline $\begin{array}{l}\text { Concentration } \\
\text { des prix }\end{array}$ & $\begin{array}{l}\text { Somme (Spécialisations dans } \\
\text { chaque grand prix français) }{ }^{2} \text {. }\end{array}$ & $\begin{array}{l}\text { Partenariats } \\
\text { français }\end{array}$ & $\begin{array}{l}\text { Ln }(1+n b \text { de parte- } \\
\text { nariats français cités). }\end{array}$ \\
\hline Prix étrangers & $\begin{array}{l}\text { Ln }(1+\text { nb de prix étrangers } \\
\text { cités). }\end{array}$ & Statut juridique & $\begin{array}{l}\text { Société anonyme }=1 \\
\text { Sinon }=0\end{array}$ \\
\hline Diversité sectorielle & $\begin{array}{l}\text { Herfindhal }=1- \\
(\text { Somme }(\text { Spécialisation dans } \\
\left.\text { chaque secteur })^{2}\right) .\end{array}$ & Taille & $\operatorname{Ln}(1+$ effectif $)$ \\
\hline $\begin{array}{l}\text { Institutions } \\
\text { françaises }\end{array}$ & $\begin{array}{l}\text { Ln }(1+n b \text { de relations avec des } \\
\text { institutions françaises })\end{array}$ & $\hat{A} g e$ & $\begin{array}{l}\text { Ln(1+(2002-Date } \\
\text { d'observation). }\end{array}$ \\
\hline Clients réputés & $\begin{array}{l}\text { Ln }(1+(\text { nb de clients du CAC40 } \\
+ \text { nb de leaders français+ nb de } \\
\text { leaders internationaux })\end{array}$ & $\begin{array}{l}\text { Dimension } \\
\text { internationale }\end{array}$ & $\begin{array}{l}\mathrm{Si} \ll \text { oui } »=1, \\
\mathrm{Si} \ll \text { non } »=0\end{array}$ \\
\hline
\end{tabular}

\begin{tabular}{|l|c|c|c|c|c|}
\hline \multicolumn{7}{|c|}{ Tableau 4 } \\
DESCRIPTION DES VARIABLES \\
\hline \multirow{2}{*}{ Nom des variables } & $\begin{array}{c}\text { Nombre } \\
\text { individus }\end{array}$ & Min. & Max. & Moyenne & Écart type \\
\hline Concentration des prix & 87 & 0 & 1 & 1,69 & 0,29 \\
\hline Prix étrangers (ln) & 87 & 0 & 3,52 & 0,202 & 0,57 \\
\hline Diversité sectorielle & 87 & 0 & 1 & 0,28 & 0,17 \\
\hline Institutions françaises (ln) & 87 & 0 & 1,79 & 0,25 & 0,44 \\
\hline Clients réputés (ln) & 87 & 0 & 3,09 & 1,82 & 0,81 \\
\hline Partenariats français (ln) & 87 & 0 & 1,38 & 0,09 & 0,307 \\
\hline Statut juridique & 87 & 0 & 1 & 0,66 & 0,47 \\
\hline Taille (ln) & 87 & 0,69 & 5,33 & 2,85 & 1,04 \\
\hline Âge (ln) & 87 & 0,69 & 3,74 & 2,47 & 0,69 \\
\hline Dimension internationale & 87 & 0 & 1 & 0,32 & 0,47 \\
\hline
\end{tabular}


que le statut juridique et la dimension internationale n'expliquent aucun des trois indicateurs retenus pour mesurer la performance créative. Cependant, l'âge influence positivement les prix étrangers et la diversité sectorielle. Cela signifie alors que plus une agence est expérimentée, plus elle participe à des concours étrangers, où elle est récompensée, et plus elle travaille avec des clients appartenant à des secteurs industriels variés. Au fil du temps, l'agence étend alors ses relations vers l'étranger et vers des horizons industriels divers.

En revanche, la taille influe négativement la performance créative mesurée par la diversité sectorielle. Plus une agence est de grande taille, moins son portefeuille commercial comprend des clients de secteurs différents. Ce résultat se comprend en tenant compte de l'investissement important des agences pour réaliser un diagnostic concurrentiel d'un marché et pour appréhender les logiques institutionnelles en vigueur sur ce marché en vue de proposer un nouveau produit ou service. Or, une agence de grande taille a un souci de rentabilité plus exacerbé: elle doit décrocher de nombreux mandats pour couvrir ses frais fixes. Dans ce sens, il lui est plus avantageux de travailler pour des clients dont les activités sont proches.

Concernant l'hypothèse 1 , nous constatons que la performance créative d'une organisation est une fonction croissante de la multiplicité de ses relations avec les institutions étatiques et professionnelles nationales. Il est difficile de valider l'hypothèse 1 , car nous constatons que les résultats diffèrent selon l'indicateur retenu. En effet, si les relations de l'agence avec des institutions françaises expliquent positivement la performance créative mesurée par la concentration des récompenses citées par rapport aux grands prix français, elles caractérisent négativement la diversité sectorielle de l'agence. Toutefois, en raison des seuils de

\begin{tabular}{|l|c|c|c|c|c|c|c|c|c|c|}
\hline \multicolumn{10}{|c|}{ Tableau 5 } \\
\multicolumn{10}{|c|}{ CORRÉLATIONS ENTRE LES VARIABLES DE L'ÉTUDE } \\
\hline & 1 & 2 & 3 & 4 & 5 & 6 & 7 & 8 & 9 & 10 \\
\hline $\begin{array}{l}\text { 1) Concentration } \\
\text { des prix }\end{array}$ & 1 & & & & & & & & & \\
\hline 2) Prix étrangers (ln) & $-0,118$ & 1 & & & & & & & & \\
\hline 3) Diversité sectorielle & $-0,119$ & $-0,067$ & 1 & & & & & & & \\
\hline 4) Instit. françaises (ln) & $0,384^{* *}$ & 0,146 & $-0,222^{*}$ & 1 & & & & & & \\
\hline 5) Clients réputés (ln) & 0,145 & 0,131 & $-0,303^{* *}$ & 0,169 & 1 & & & & & \\
\hline $\begin{array}{l}\text { 6) Partenariats } \\
\text { français (ln) }\end{array}$ & $-0,034$ & $-0,107$ & $-0,195$ & $-0,09$ & 0,172 & 1 & & & & \\
\hline 7) Statut juridique & 0,024 & 0,170 & 0,007 & $0,241^{*}$ & 0,051 & $-0,142$ & 1 & & & \\
\hline 8) Taille (ln) & 0,183 & 0,071 & $-0,194$ & $0,212^{*}$ & $0,231^{*}$ & $-0,089$ & $0,289^{* *}$ & 1 & & \\
\hline 9) Âge (ln) & 0,166 & $0,229^{*}$ & 0,085 & 0,004 & 0,169 & 0,012 & 0,205 & $0,409^{* * *}$ & 1 & \\
\hline 10) Dim. internationale & $-0,085$ & 0,172 & 0,060 & 0,031 & 0,086 & $-0,041$ & 0,189 & 0,080 & 0,074 & 1 \\
\hline
\end{tabular}

Remarque : corrélation significative bilatérale à $1 \%(* *)$ et à $5 \%(*)$. 


\begin{tabular}{|c|c|c|c|c|c|c|c|c|c|c|c|c|}
\hline \multicolumn{13}{|c|}{$\begin{array}{c}\text { Tableau } 6 \\
\text { MODÈLES STATISTIQUES }\end{array}$} \\
\hline & \multicolumn{4}{|c|}{ Concentration des prix } & \multicolumn{4}{|c|}{ Prix étrangers } & \multicolumn{4}{|c|}{ Diversité sectorielle } \\
\hline & Base & H1 & $\mathrm{H} 2$ & $\mathrm{H} 3$ & Base & H1 & $\mathrm{H} 2$ & $\mathrm{H} 3$ & Base & H1 & $\mathrm{H} 2$ & $\mathrm{H} 3$ \\
\hline Instit. françaises & & 0,398 **** & 0,390 *** & $0,391 * *$ & & 0,139 & 0,127 & 0,129 & & $-0,191 *$ & $-0,151$ & $-0,148$ \\
\hline Clients réputés & & & 0,052 & 0,063 & & & 0,078 & 0,102 & & & $-0,274 * *$ & $-0,241^{* *}$ \\
\hline Partenaires français & & & & $-0,059$ & & & & $-0,121$ & & & & $-0,169 *$ \\
\hline Variables de contrôle & & & & & & & & & & & & \\
\hline Statut & $-0,025$ & $-0,108$ & $-0,105$ & $-0,112$ & 0,117 & 0,087 & 0,093 & 0,078 & 0,040 & 0,080 & 0,061 & 0,040 \\
\hline Taille & 0,150 & 0,069 & 0,061 & 0,053 & $-0,064$ & $-0,092$ & $-0,105$ & $-0,120$ & $-0,288 * *$ & $*-0,250 * *$ & $-0,203 *$ & $-0,224 *$ \\
\hline Âge & 0,117 & 0,165 & 0,160 & 0,163 & $0,221 *$ & $0,238^{* * *}$ & $0,230 *$ & $0,237 * *$ & $0,190 *$ & 0,167 & $0,197 *$ & $0,206^{*}$ \\
\hline Dim. internationale & $-0,101$ & $-0,094$ & $-0,098$ & $-0,100$ & 0,139 & 0,141 & 0,135 & 0,132 & 0,061 & 0,058 & 0,078 & 0,073 \\
\hline R2 & 0,055 & 0,198 & 0,201 & 0,204 & 0,090 & 0,107 & 0,113 & 0,127 & 0,076 & 0,109 & 0,178 & 0,205 \\
\hline $\mathrm{F}$ & & 4,007 *** & $3,349 * *$ & $2,892 *$ & & $1,945^{*}$ & 1,697 & 1,639 & & $1,985^{*}$ & $2,883 * *$ & $2,904 * * *$ \\
\hline
\end{tabular}

Remarque : corrélation significative bilatérale à $1 \%(* * *)$, à $5 \%(* *)$ et $10 \%(*)$.

significativité, nous pouvons dire que les relations de l'agence avec des institutions expliquent la performance créative professionnelle. Si Amabile (1988) explique qu'un nombre important de contraintes nuit à la créativité, et donc à la performance créative des organisations, nous constatons que, dans le cas des agences de design, cela n'est pas confirmé au niveau de l'environnement institutionnel. Ce résultat confirme un aspect de la définition du métier de designer: être capable de gérer de multiples contraintes en même temps (Borja de Mozota, 2002).

À propos de l'hypothèse 2, les tests statistiques signalent que la performance créative d'une organisation est une fonction décroissante de la multiplicité de ses relations avec des clients réputés. La variable « clients réputés » explique négativement la performance créative mesurée par la diversité sectorielle: 1'hypothèse 2 est donc sou- tenue. Plus une agence de design travaille pour des clients réputés, moins elle exprime sa performance créative auprès d'entreprises de secteurs variés. De ce fait, une agence associée à ce type de clients a moins de liberté dans sa créativité. L'analyse d'Amabile (1988) s'avère alors exacte au niveau de l'environnement marchand: la créativité des agences de design est entravée par les exigences des clients réputés. Enfin, la performance créative d'une organisation est une fonction décroissante de la multiplicité de ses relations avec les partenaires nationaux. Les analyses précisent que les partenariats français caractérisent négativement la capacité de l'agence à travailler avec des clients de secteurs différents: l'hypothèse 3 n'est pas soutenue. Les agences de design gagnent sûrement en richesses en faisant appel à d'autres professionnels (Drazin et al., 1999), mais le sentiment d'être contrôlées, voire épiées dans 
leur créativité, les freinent dans la création de nouvelles idées valorisables et utiles (Amabile et al., 1996).

Ces différents résultats nous amènent à constater des influences certaines entre les institutions françaises, les clients réputés et les partenaires français et la performance créative des agences. Ils nous conduisent également à distinguer la valorisation et l'utilité attribuée, d'une part, par les clients et les partenaires et, d'autre part, par les institutions étatiques et professionnels. Ainsi, l'environnement des agences de design ne peut pas être réduit à une seule entité: il doit être différencié en fonction des acteurs impliqués.

L'hypothèse 2 nous amène à nous interroger sur la place des clients moins réputés dans le champ organisationnel du design industriel. Si les agences introduisent de la créativité dans les secteurs de leurs clients (ministère de l'Industrie, 2002; Walsh, 2000), peuvent-elles profiter également sur le plan créatif de leurs collaborations avec des clients? Une émulation créative se produirait alors entre les clients non réputés et les agences de design.

En outre, sur le plan managérial, il serait intéressant d'étudier les échanges entre les agences et les clients non réputés pour comprendre les procédures d'émulations créatives : les projets de court terme profitent-ils sur le plan créatif à l'agence de design? Tous les types de projets en design industriel favorisent-ils les échanges créatifs? Est-ce que l'échange créatif est plus important avec un client ayant un designer intégré ? Ces réponses pourront soutenir le développement stratégique des agences de design sur le plan créatif et les aider à remplir leur mission première: introduire de la créativité dans l'environnement écono- mique et culturel (Walsh, 2000; Hetzel, 1995).

\section{CONCLUSION}

La question initiale de l'article était d'expliquer l'influence des relations interorganisationnelles sur la performance créative des organisations en adoptant l'idée selon laquelle les institutions ne sont pas uniquement des « cages de fer ». Ce travail s'avère donc un apport aux théories néoinstitutionnalistes, en illustrant le concept de Washington et Ventresca (2004) par une étude sur le champ du design industriel en France. Cette recherche montre aussi que si certaines relations interorganisationnelles inhibent la performance créative des agences, d'autres la soutiennent. Ces conclusions enjoignent alors les agences de design à analyser l'impact de leurs relations sur la caractéristique fondamentale de leur métier, à savoir leur créativité. Si les agences veulent travailler avec des clients réputés pour gagner en prestige, force est de reconnaître l'impact négatif sur leur performance créative.

Cette étude n'est pas, cependant, exempte de limites. En effet, la difficulté à définir ce qui est valorisable et utile dans la créativité des organisations nous amène à choisir des indicateurs, où les jugements subjectifs de professionnels et d'industriels sont impliqués. Cela explique sans doute pourquoi certains résultats sont différents selon les indicateurs, notamment pour l'hypothèse 1 . De plus, les données étudiées sont renseignées par les agences de design elles-mêmes : même si leur description est vraie, elle est contextualisée et orientée vers la recherche de nouveaux clients. 
Néanmoins, cette étude soulève des questions théoriques, notamment sur la conjonction des pressions isomorphiques et hétéromorphiques des institutions. L'existence de telles institutions pourraient expliquer le fait que, seuls, certains champs organisationnels accueillent de la diversité (Lounsbury, 2001). En revanche, est-ce que seuls des champs organisationnels construits autour de pratiques créatives, comme le design industriel, la cuisine, la publicité, l'architecture, accueillent de telles institutions? Eu égard à l'étude menée par Washington et al. (2004) sur l'intégration de nouveaux programmes sportifs dans les universités américaines, il ne semble pas. Mais, peu de recherches ont été réalisées avec leur approche théorique.

De plus, si les institutions encouragent la remise en question des normes, les organisations divergentes ne sont plus des renégats (Kondra et al., 1998) mais des entités légitimes. Que signifie alors la classification d'Oliver (1991) ? Comment distinguer une organisation guidée par le soutien insti- tutionnel vers des stratégies émergentes et la critique des normes, d'une autre en opposition avec les normes institutionnelles en vigueur? Ces questions méritent d'être approfondies: elles enrichiront les théories néoinstitutionnalistes par une approche alternative aux institutions en tant que « cages de fer ».

Enfin, cet article s'est focalisé sur l'impact de trois acteurs de l'environnement des organisations sur la performance créative des organisations, à savoir les institutions étatiques et professionnelles nationales, les clients réputés et les partenaires professionnels nationaux. Malgré les conclusions de Greve et Taylor (2000), il serait intéressant de s'inquiéter de l'influence de ces mêmes entités au plan international, mais également d'intégrer l'impact des clients non réputés sur la performance créative. Ces recherches futures permettront ainsi de poursuivre l'approfondissement de l'étude des changements organisationnels en management stratégique.

\section{BibliograPhie}

Amabile T., "A model of creativity and innovation in organizations", Organizational Behavior, vol. 10, 1988, p. 123-167.

Amabile T., Conti R., Coon H., Lezenby J., Herron M., “Assessing the work environment for creativity", Academy of Management Journal, vol. 39, n 5, 1996, p. 1154-1184.

Atamer T., Durand R., Reynaud E., « Développer l'innovation », Dossier, Revue française de gestion, $\mathrm{n}^{\circ} 155,2005$, p. 13-21.

Baker W. E., Faulkner R. R. et Fisher G. A., "Hazards of the market: the continuity and dissolution of interorganizational market relationships", American Sociological Review, april, vol. 63, n² 2, 1998, p. 147-177.

Bejamin B. A. et Podolny J. M., "Status, Quality and Social Order in California wine industry", Administrative Science Quarterly, vol. 44, n³, 1999, p. 563-589.

Borja de Mozota B., Design management, Éditions d'Organisations, 2002. 
de Noblet J., Design. Le Geste et le Compas, Éditions Aimery Somogy, Paris, 1988.

Desreumaux A., « Théorie néo-institutionnelle, management stratégique et dynamique des organisations », Institutions et gestion, Huault I. (coord.), éd. Vuibert, Paris, 2004, p. 29-47. DiMaggio P. J., Powell W.W., “The iron cage revisited: institutional isomorphism and collective rationality in organisational fields", American Sociological Review, 48, 1983, p. 174-160.

Drazin R., Glynn M-A., Kazanjian R., "Multilevel theorizing about creativity in organizations: a sense-making perspective", Academy of Management Review, vol. 24, n 2, 1999, p. $286-307$

Durand R., Rao H., Monin P., "Code and conduct in French cuisine: impact of code-changes on external evaluations”, Working paper; HEC Paris, 2005.

Edelman L. B., Uggen C., Erlanger H., "The endogeneity of legal regulation: grievance procedures as rational myth", The American Journal of Sociology, vol. 105, $\mathrm{n}^{\circ} 2$, September 1999, p. 406-454.

Elsbach K. D, "Managing organizational legitimacy in the California Cattle Industry: The construction and effectiveness of verbal accounts", Administrative Science Quarterly, 39, 1994, p. 57-88.

ENDTE, «Design : du "cosmétique" au stratégique », Revue française de gestion, septembre-octobre 1990, p. 94-100.

Farjoun M., "The dialectics of institutional development in emerging and turbulent fields: the history of pricing conventions in the on-line database industry", Academy of Management Journal, vol. 45, $\mathrm{n}^{\circ}$ 5, 2002, p. 848-874.

Fombrun C., Shanley M., "What's in a name? Reputation building and corporate strategy", Academy of Management Journal, vol. 33, 2, 1990, p. 233-258.

Ford C., "A theory of individual action in multiple social domains", Academy of Management Review, vol. 21, n 4, 1996, p. 1112-1142.

Gerber T., "Structural Change and post-socialist stratification: labor market transitions in contemporary Russia”, American Sociological Review, vol. 67, n 5, 2002, p. 629-659.

Greve H., Taylor A., "Innovations as catalysts for organizational change: shifts in organizational cognition and search", Administrative Science Quarterly, 45, 2000, p. 54-80.

Guidot R., L’histoire du design 1940-2000, éditions Hazan (réédition), Paris, 2000.

Hargadon A., Douglas Y., "When innovations meet institutions: Edison and the design of the electric light”, Administrative Science Quarterly, 46, 2001, p. 476-501.

Hetzel P., « Pour renouveler les processus d'innovation en entreprise », Revue française de gestion, $\mathrm{n}^{\circ} 103$, mars-mai 1995, p. 87-98.

Hoffman A., "Institutional evolution and change: environment and the U.S. chemical industry", Academy of Management Journal, 42, 4, 1999, p. 351-371.

Jones C., Manev I., Foster P., "Legitimation through differentiation? How architects socially construct their reputations", workshop, 2001.

King A. A., Lenox M. J., "Industry self-regulation without sanctions: the chemical industry's responsible care program", Academy of Management Journal, vol. 43, $\mathrm{n}^{\circ}$ 4, 2003, p. 698-716. 
Kondra A. Z. et Hinings C. R., "Organizational diversity and change in institutional theory", Organization Studies, vol. 19, n 5, 1998, p. 743-767.

Lounsbury M., "Institutional sources of practice variation: staffing college and university recycling programs", Administrative Science Quarterly, 46, 2001, p. 29-56.

Lucie-Smith E., A history of industrial design, éditions Phaidon-Oxford, 1983.

Meyer C., La stratégie design de l'entreprise, Les Échos-Études, coll. «Le management stratégique », 2001.

Meyer J., Rowan B., "Institutionalized organisations: formal structure as myth and ceremony", American Journal of Sociology, 83, 1977, p. 340-367.

Milliken F., Martins L. et Morgan H., "Explaining organizational responsiveness to workfamily issues: the role of human resource executives as issue interpreters", Academy of Management Journal, October, vol. 41, n 5, 1998, p. 580-592.

Ministère de l'Économie, des Finances et de l'Industrie, Les pratiques du design en PMI, Rapport d'étude, DesignFrance et Tremplin Protocoles, novembre 2002.

Misruchi et Fein, "The social construction of organizational knowledge: a study of uses of coercitive, mimetic and normative isomorphism", Administrative Science Quarterly, 44, 1999, p. 653-683.

Oliver C., "Strategic responses to institutional processes", Academy of Management Review, vol. 16, n 1, 1991, p. 145-179.

Pfeffer J. et Salancik G., The external control of organizations, Harper et Row Publishers, New York, 1978.

Rao H., Monin P., Durand R., "Institutional change in Toque Ville: Nouvelle Cuisine as an identity movement in French Gastronomy”, American Journal of Sociology, vol. 108, $\mathrm{n}^{\circ} 4$, 2003, p. 795-843.

Revue française de gestion, « Développer l'innovation », numéro spécial, vol. 31, n 155, mars/avril 2005.

Schoonhoven, Eisenhardt, Lyman, "Speeding products to market: Waiting time to first product introduction in new firms", Administrative Science Quarterly, 35, 1990, p. 177-207.

Scott W. R., Institutions and Organizations, $2^{\text {nd }}$ édition (1994 première édition, Foundations for organizational science), A sage publications series, 2001.

Selznick P. K., Leadership in administration, Evanston, Ill., Row, Petersonet Co, 1957.

Suchmann M., "Managing legitimacy: strategic and institutional approaches", Academy of Management Review, vol. 20, n 3, 1995, p. 571-610.

Thietart R.-A., Méthodes de Recherche en Management, Dunod, Paris, 2003, p. 225-256 (chap. 9), p. 257-287 (chap. 10).

Van den Bulte C. et Lilien G., "Medical innovation revisited: social contagion versus marketing effort", The American Journal of Sociology, vol. 106, n 5, March 2001, p. 14091435.

Walsh V., "Design, Innovation, and the boundaries of the firm", Design Management Journal Academic Review, 2000, p. 74-92. 
76 Revue française de gestion $-\mathrm{N}^{\circ} 164 / 2006$

Washington M. et Ventresca M., "How organizations change: the role of institutional support mechanisms in the incorporation of higher education visibility strategies: 1874-1995", Organization Science, vol. 15, $\mathrm{n}^{\circ}$ 1, 2004, p. 82-97.

Woodman R., Sawyer J., Griffin R., "Toward a theory of organizational creativity”, Academy of Management Review, vol. 18, $\mathrm{n}^{\circ}$ 2, 1993, p. 93-321.

Zuckerman E., "The categorical imperative: securities analysts and the illegitimacy discount", American Journal of Sociology, vol. 104, n 5, 1999, p. 398-1438. 